\title{
Estudio etnobotánico, arquitectura foliar y anatomía vegetativa de Agastache mexicana ssp. mexicana y A. mexicana ssp.xolocotziana
}

\author{
Etnobotany, leaf architecture, and vegetative anatomy of Agastache mexicana ssp. mexicana \\ and $A$. mexicana ssp. xolocotziana
}

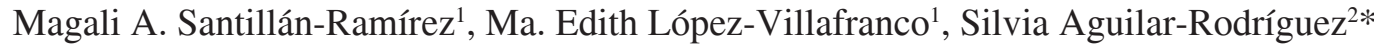 \\ y Abigail Aguilar-Contreras ${ }^{3}$ \\ ${ }^{1}$ Herbario IZTA, FES Iztacala, Universidad Nacional Autónoma de México. Apartado postal 314, 54090, Tlalnepantla, Estado de México, México \\ ${ }^{2}$ Laboratorio de Botánica, UMF, FES Iztacala, Universidad Nacional Autónoma de México, Apartado postal 314, 54090, Tlalnepantla, Estado de \\ México, México \\ ${ }_{3}^{3}$ Herbario Medicinal del Instituto Mexicano del Seguro Social (IMSSM), Avenida Cuauhtémoc 330, Colonia Doctores, 06725, México, D.F. \\ *Correspondencia: siagro@campus.iztacala.unam.mx
}

\begin{abstract}
Resumen. El presente trabajo incluye información etnobotánica, así como la arquitectura foliar y anatomía vegetativa de Agastache mexicana ssp. mexicana (toronjil morado) y A. mexicana ssp. xolocotziana (toronjil blanco), con la finalidad de que sean incluidas en la Farmacopea Herbolaria de los Estados Unidos Mexicanos (FHEUM) y que sus caracteres anatómicos se utilicen para validar la identidad del material vegetal en el control de calidad. El registro de la información etnobotánica se efectuó por medio de entrevistas semiestructuradas a pobladores del municipio de Temoaya, Estado de México, México. Para el estudio anatómico y arquitectura foliar, se elaboraron preparaciones semipermanentes y permanentes de tallos y hojas. Ambos toronjiles son utilizados por los habitantes de la zona de estudio como recurso terapéutico y de ornato; los distinguen por la forma de las hojas, el color de la flor y el aroma, pero no existen preferencias de uso entre ambos. La arquitectura foliar sigue el patrón acródromo de posición basal con un desarrollo imperfecto; las areolas muestran un desarrollo imperfecto con disposición al azar, con vénulas simples o ramificadas una vez y el margen con dientes cunonioides en ambas especies. Diferencias anatómicas se observan en el tallo y son los tricomas no glandulares uniseriados en mayor abundancia y las bandas de esclerénquima más desarrolladas los caracteres que distinguen a $A$. mexicana ssp. xolocotziana de A. mexicana ssp. mexicana.
\end{abstract}

Palabras clave: Agastache, etnobotánica, arquitectura foliar, anatomía, Temoaya.

\begin{abstract}
This work provides information on ethnobotany, leaf architecture and anatomy of Agastache mexicana ssp. mexicana (toronjil morado) and A. mexicana ssp. xolocotziana (toronjil blanco), with the purpose that they will be included in the Pharmacopeia Herbolaria of the Estados Unidos Mexicanos (FHEUM) and that their anatomical characters could be used to validate the identity of the botanical material in quality control. We gathered ethnobotanical information using semi-structured interviews of people from Temoaya, Mexico State, Mexico. Semi-permanent and permanent slides for structural analysis and leaf architecture were made. Both toronjiles are used by the inhabitants as a therapeutic and ornamental resource, they are distinguished by the leaf shape, flower color, and flavor, but there are no use preferences between them. Leaf architecture follows the acrodromous pattern of basal position with an imperfect development; areoles show an imperfect development with a random arrangement, with simple or once-branched veinlets and the margin with cunonioid teeth in both species. Anatomical differences are observed in the stem include the more abundant uniseriate non- glandular trichomes and the more highly developed developed sclerenchyma bands in A. mexicana ssp. xolocotziana with respect to $A$. mexicana ssp. mexicana.
\end{abstract}

Key words: Agastache, ethnobotany, leaf architecture, anatomy, Temoaya.

\section{Introducción}

En México se han desarrollado diferentes estudios etnobotánicos, siendo los de plantas medicinales los más

Recibido: 08 mayo 2007; aceptado: 22 enero 2008 numerosos (Martínez, 1994); éstas forman parte importante de los recursos terapéuticos que emplea la medicina tradicional popular mexicana y que han representado una alternativa para la salud de los diferentes grupos étnicos del país (Aguilar y Camacho, 1985; Toledo, 1995); entre los que se encuentran los otomíes y mestizos que habitan 
en Temoaya, Estado de México. La población cuenta con el mayor número de habitantes otomíes, representantes de una cultura que aún mantiene viva sus tradiciones y usa frecuentemente sus recursos vegetales. De acuerdo con su saber médico tradicional, los habitantes de Temoaya consideran 2 tipos de causa de enfermedades: naturales y sobrenaturales; en éstas últimas, conocidas como síndromes de filiación cultural (SFC) Campos, 1997), el padecimiento además de incluir aspectos físicos, expresa elementos propios de su cultura. A través de los estudios llevados a cabo con diferentes grupos humanos del país, se ha observado que la curación de estas nosologías se vincula con la fe y con la intervención de terapeutas tradicionales, los cuales siguen haciendo uso de plantas medicinales para curar los SFC, entre otros el susto o espanto (Instituto Nacional Indigenista, 1995).

En diferentes poblaciones de México, las plantas comúnmente utilizadas para curar el susto son el toronjil morado, Agastache mexicana (Kunth) Lint et Epling ssp. mexicana Bye, Linares et Ramamoorthy, y el blanco, Agastache mexicana (Kunth) Lint et Epling ssp. xolocotziana Bye, Linares et Ramamoorthy, pertenecientes a la familia Lamiaceae (Aguilar et al., 1994). Ambas subespecies se han estudiado desde el punto de vista farmacológico (Galindo, 1982; Contreras, 1987), quimiotaxonómico (Espíritu, 1991; Estrada-Reyes et al., 2004), fitoquímico (Aguirre, 2003) y de propagación (Chávez, 1986), pero para el municipio de Temoaya, sólo se cuenta con investigaciones epidemiológicas (Medina, 1998) y antropológicas (Cervantes, 1978).

Los toronjiles son plantas herbáceas que crecen en los bosques de pino-encino y en los huertos familiares de la comunidad en estudio. Se reconocen como especies de uso frecuente dentro de la terapéutica tradicional en Temoaya; están registradas en las monografías de la Extrafarmacopea Herbolaria de los Estados Unidos Mexicanos, pero todavía no cuentan con los estudios requeridos, entre ellos los anatómicos, para ser integradas en la Farmacopea Herbolaria de los Estados Unidos Mexicanos (FHEUM) (FEHUM, 2001). En este trabajo se registró la información etnobotánica de $A$. mexicana ssp. xolocotziana y A. mexicana ssp. mexicana en el municipio de Temoaya, Estado de México, México y se realizaron los estudios sobre la arquitectura foliar y la anatomía de hoja y tallo de estas dos subespecies, con la finalidad de aportar dicha información a la FHEUM y señalar si existen caracteres estructurales vegetativos que contribuyan a separar ambas subespecies.

\section{Material y métodos}

Área de estudio. El Municipio de Temoaya se localiza en la porción centro norte del Estado de México, México; a $\operatorname{los} 19^{\circ} 28^{\prime} 50^{\prime \prime} \mathrm{N}$ y $99^{\circ} 36^{\prime} 12^{\prime \prime} \mathrm{O}$ y a una altitud de 2750 m snm; el clima es templado subhúmedo, con lluvias durante el verano; la temperatura media anual de $13.4{ }^{\circ} \mathrm{C}$, teniendo una precipitación pluvial de 899.2 mm (Arzate, 1999; García, 2004). El tipo de vegetación corresponde a un bosque de pino-encino (Rzedowski, 1988). Los árboles y arbustos que caracterizan la vegetación pertenecen a los géneros Pinus, Quercus, Abies, Taxodium, Juniperus, Baccharis, Fuchsia, Satureja y Symphoricarpos. En el estrato herbáceo predominan especies de las familias: Asteraceae, Poaceae, Fabaceae, Lamiaceae, Scrophulariaceae y Rosaceae, entre otras. Esta comunidad vegetal ha sufrido alteraciones debido al incremento demográfico y al establecimiento de terrenos para agricultura y ganadería.

En la zona de estudio la familia constituye la unidad básica de la organización social. La economía está basada principalmente en el comercio y la agricultura. Existen diferentes centros educativos en gran parte de las comunidades, y la alimentación, el ambiente, la vivienda y la educación son algunos de los factores que influyen en su salud. Según registros, el $16.3 \%$ de la población está afiliada al Instituto Mexicano del Seguro Social (IMSS) o al Instituto de Seguridad y Servicios Sociales de los Trabajadores del Estado (ISSSTE), mientras que $83.7 \%$ acude a otras instancias de salud como son el Instituto de Salud del Estado de México (ISEM), el centro de Desarrollo Integral de la Familia (DIF), o a las clínicas particulares y consultorios médicos. De igual importancia en Temoaya, es el grupo de promotoras de salud ÑHAHTO, que emplea como recurso médico las plantas medicinales y algunos otros productos galénicos.

Con base en las propuestas de Martínez (1976), Hernández-Xolocotzi (1979) y Cotton (1998), la información etnobotánica se registró realizando entrevistas semiestructuradas a la población en general, así como a los especialistas en salud (médicos alópatas y médicos tradicionales populares) de la zona en estudio; para obtener la información se mostraron ejemplares herborizados de los diferentes toronjiles a los habitantes entrevistados. La recolecta de los toronjiles y el trabajo de herbario se llevaron a cabo siguiendo los lineamientos de Lot y Chiang (1986). Los ejemplares de referencia se encuentran depositados en la colección etnobotánica del Herbario IZTA de la FES-Iztacala de la UNAM, con los números de registro 866 y 867 (Fig. 1) y en el Herbario IMSSM, del Instituto Mexicano del Seguro Social están integrados con los números de registro 14747 y 14748 . El trabajo de gabinete consistió en la elaboración del cuestionario para realizar las entrevistas semiestructuradas y en la captura de las mismas en una base de datos (BD: Toronjil), la cual se 

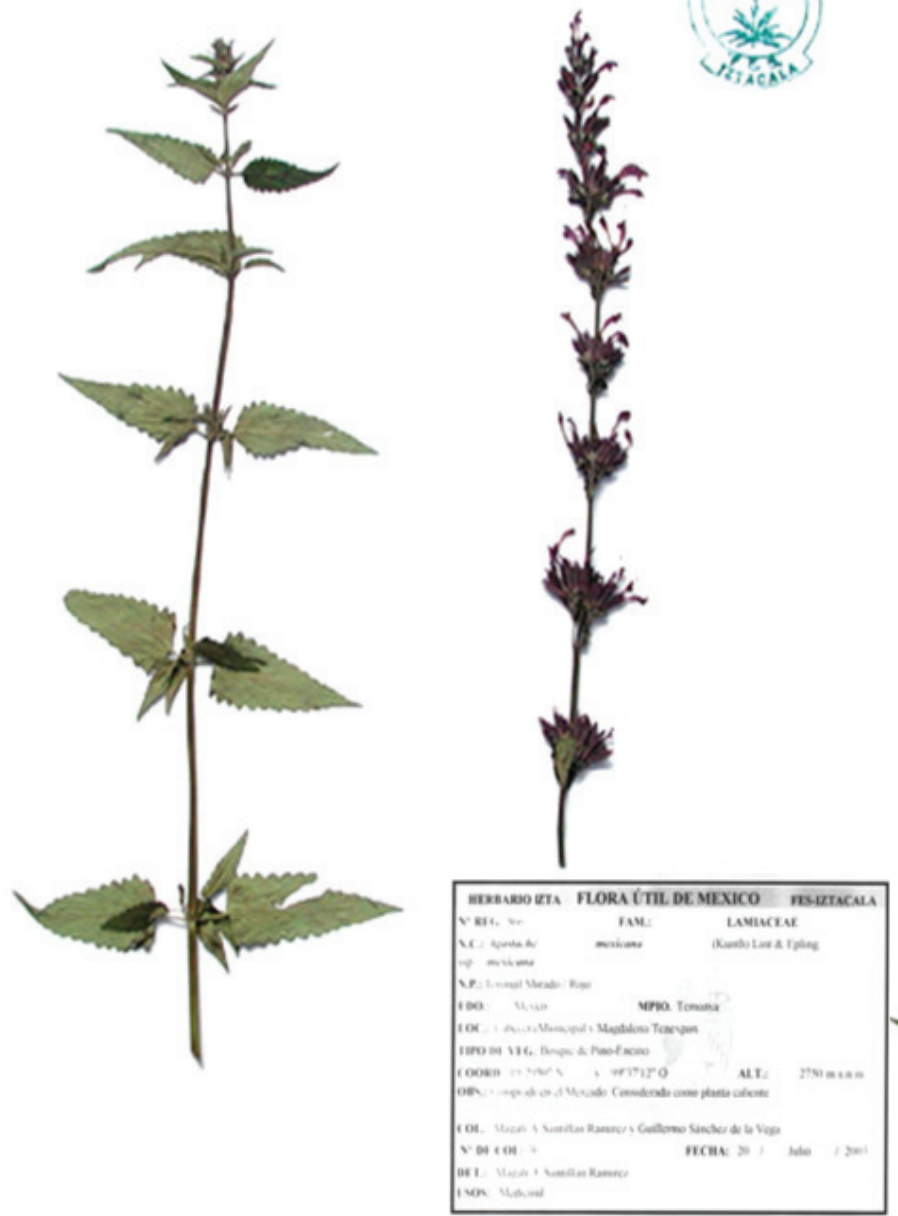

Figura 1. Ejemplares de referencia de toronjil morado (Núm. 866) y toronjil blanco (Núm. 867) depositados en el Herbario IZTA.

integró a la base de información etnobotánica del Herbario IZTA. Para distinguir las 2 subespecies de toronjil se obtuvieron caracteres morfológicos, como tamaño del individuo, largo y ancho de hoja, color de cáliz y corola de diferentes ejemplares depositados en ambos herbarios.

Para describir la arquitectura foliar y la anatomía vegetativa, se recolectaron 8 plantas de $A$. mexicana ssp. mexicana y $A$. mexicana ssp. xolocotziana en el municipio de Temoaya. El material se fijó en FAA (formol 5 ml, ácido acético glacial $5 \mathrm{ml}$, y alcohol etílico al $70 \% 90 \mathrm{ml}$ ). Con el propósito de evidenciar la organización de las areolas y dientes en los márgenes, las hojas completas se aclararon con $\mathrm{NaOH}$ al $10 \%$, calentando a una temperatura alta pero sin hervir, hasta extraer el contenido celular; en seguida se lavaron con agua y se agregó cloro comercial al $30 \%$ hasta

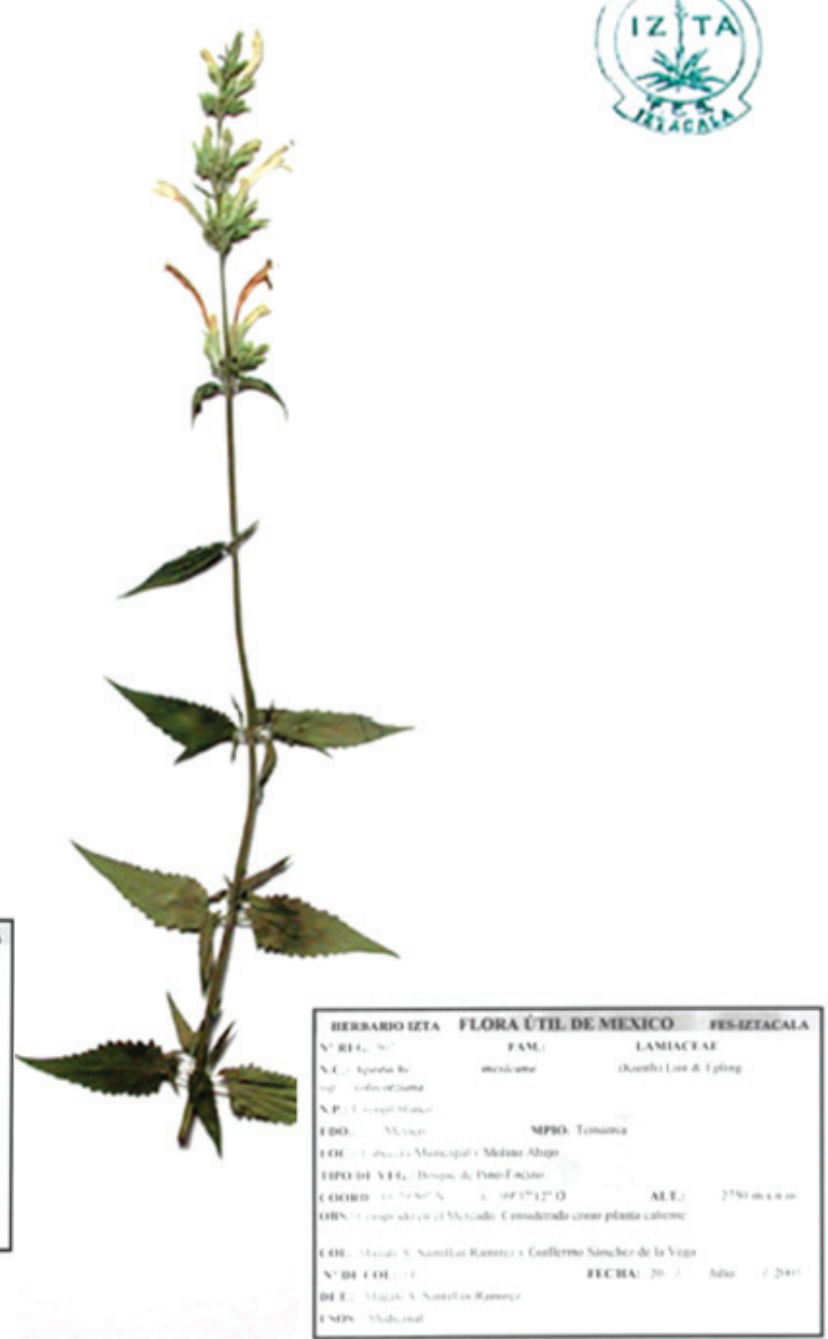

que las hojas se blanquearon; a continuación se enjugaron y tiñeron con safranina acuosa; se deshidrataron en una serie de alcoholes graduales; se terminaron de deshidratar en xilol y finalmente se montaron en resina sintética (Triplett y Kirchoff, 1991; Aguilar-Rodríguez, 1998; Murillo, 2001). Las descripciones se hicieron de acuerdo con la terminología propuesta por Hickey $(1973,1974)$. Para el trabajo anatómico, la parte media de la lámina foliar, así como secciones de la porción basal, media y superior del tallo se fijaron en FAA durante 48 horas. La obtención de las muestras en los 3 niveles del tallo se llevó a cabo para reconocer alguna variación anatómica en el mismo. Posteriormente se sometieron a deshidratación en alcoholes etílicos graduales, se deshidrataron con xilol y se incluyeron en parafina histológica a $58-60{ }^{\circ} \mathrm{C}$ (Curtis, 
1986; Aguilar-Rodríguez, 1998). Se obtuvieron secciones transversales de $15 \mu \mathrm{m}$ de grosor empleando un micrótomo de rotación. Después se siguió la técnica de tinción con safranina-verde rápido (Curtis, 1986) y se montaron en resina sintética. Algunas secciones transversales de tallo y lámina foliar se obtuvieron realizando cortes a mano con navaja de afeitar (Aguilar-Rodríguez, 1998), montándose en gelatina glicerinada coloreada con safranina; ésta es una técnica rápida, donde la tinción y el montaje son efectuados simultáneamente para evidenciar los tejidos lignificados y cutinizados. Otros cortes del plano transversal de la hoja se lograron de $16 \mu \mathrm{m}$ a $-20{ }^{\circ} \mathrm{C}$ empleando un criostato (Aguilar-Rodríguez, 1998). Se describió la anatomía de hoja y tallo de ambos toronjiles siguiendo los términos que emplean Metcalfe y Chalk (1979) y Fahn (1982). En tallos con crecimiento secundario, se cuantificó y describió el diámetro del lumen de los vasos xilemáticos con base en lo propuesto por la Asociación Internacional de Anatomistas de la Madera (IAWA Committee, 1989). Las descripciones y mediciones de los caracteres anatómicos se realizaron con un microscopio óptico marca Carl Zeizz. Se realizaron 25 mediciones de cada carácter por muestra; éstas se presentan en intervalos mínimo y máximo; los promedios del lumen de los vasos xilemáticos se registran con su desviación estándar y los valores mínimo y máximo se anotan entre paréntesis. Los dibujos de tallo y hoja se elaboraron empleando una cámara clara a $20 \times$ en un microscopio marca Nikon Labophot-2.

\section{Resultados}

Etnobotánica. Para la investigación etnobotánica sobre el conocimiento y uso de los toronjiles se entrevistaron 32 habitantes en la zona de estudio, de los cuales el $56 \%$ son mestizos y el $46 \%$ del grupo otomí. Los entrevistados fueron amas de casa (46.6\%), comerciantes (30\%), estudiantes $(10 \%)$ y servidores públicos $(13.3 \%)$. El 78 $\%$ de los entrevistados fueron mujeres.

Los habitantes de Temoaya consideran el toronjí -así nombrado por el grupo otomí-, como una planta de calidad caliente porque cura el enfriamiento: el $68 \%$ de los entrevistados, tiene conocimiento del toronjil al ser mencionado sólo por el nombre popular, mientras que el restante $32 \%$ sólo cuando se les mostró y olieron la planta dijo conocerlo. Cuando la planta no tiene flores los pobladores lo diferencian por el aroma, mencionando que el morado posee un olor dulce y el blanco un olor ácido; el $46 \%$ de la gente los reconoce por el color, el $32 \%$ por la flor y $11 \%$ por la forma de las hojas, el aroma y el sabor. Son distintas las fuentes de enseñanza del uso del toronjil como recurso médico; se registra que el $65 \%$ lo aprendió de los abuelos y los padres; $13 \%$ de los hierberos (del mercado) y $10 \%$ menciona que de "la gente" sin especificar parentesco.

Los mestizos y otomíes de la zona de estudio informan que son 2 los usos del toronjil morado y blanco: medicinal y ornamental; se emplea en el tratamiento de dolor de estómago, aire, tos, bilis, enfriamiento, vómito, nervios, pero principalmente para el susto o espanto (Fig. 2). En este estudio se identificaron varias estrategias empleadas por los habitantes de Temoaya en la prevención y curación de padecimientos; consisten en primera instancia, agotar el saber de la unidad familiar, seguida de los hierberos y curanderos y en caso de que éstos no devuelvan la salud, se recurre a las clínicas atendidas por médicos alópatas. Los resultados indican que la concepción de salud en la población puede percibirse de dos maneras diferentes, la visión popular y la visión alópata. De acuerdo al conocimiento popular, el susto se presenta cuando se ven cosas repentinas, situaciones inesperadas o por un accidente. Los signos y síntomas detectados para una persona que tiene susto son: pérdida del apetito, intranquilidad en el sueño, desánimo, pérdida de la razón y palidez. Los informantes atribuyen que la gente débil es la que se enferma de susto y se cura tomando un té de la planta de toronjil. Desde el punto de vista de la terapéutica de la medicina occidental no se registra el padecimiento, por lo tanto, no se da tratamiento alguno con medicamentos alópatas; como consecuencia, la gente al enfermarse de susto no acude a las clínicas de salud ya que enfatizan que ahí no curan eso, sólo dan calmantes.

Respecto al modo de preparación, el toronjil puede ser hervido hasta que el agua pinte, con o sin azúcar, sólo o mezclado con otras plantas como el hinojo/anojo (Foeniculum vulgare Mill.), tila (Ternstroemia pringlei (Rose) Standl.), canela (Cinnamomum zeylanicum Blume) o rosa de Castilla (Rosa centifolia L.). Otras

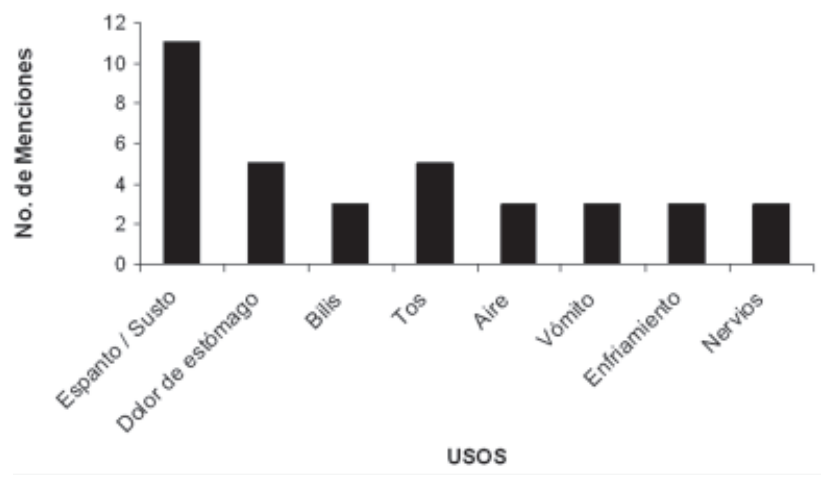

Figura 2. Usos medicinales del toronjil proporcionados por lo habitantes de Temoaya. 


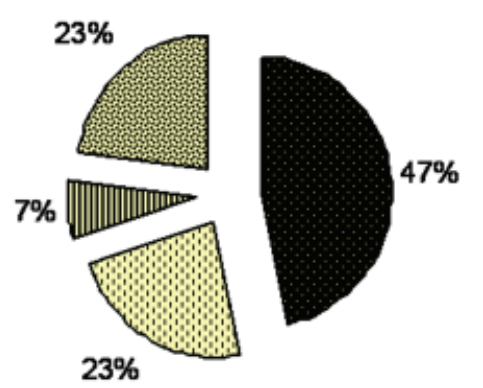

\begin{tabular}{l} 
mercado \\
Whuerto \\
W compra a ambulantes \\
monte \\
\hline
\end{tabular}

Figura 3. Lugares donde se obtiene el toronjil.

formas registradas para la preparación del toronjil fueron: a)....molido en metate, mezclado con pulque y ajenjo (Artemisia absinthium L.) para que jale el hambre; b).... en té, una ramita del blanco, el rojo, más el hinojo molido en la licuadora; c).... en té, o los dos toronjiles, anojo, hervido .... si es seco, se pone media cucharadita, se cuela y se toma por las mañanas; y d).... hervido en agua para bañar al enfermo en caso de un susto grande.

El $47 \%$ de los habitantes de Temoaya consigue el toronjil del mercado, $23 \%$ lo tiene en huertos como planta ornamental, otro $23 \%$ lo trae del monte y $7 \%$ lo obtiene comprándolo directamente de las personas que pasan a ofrecerlo en las casas (Fig. 3).

Arquitectura foliar (Fig. 4). Lámina entera, de forma ovado-lanceolada, con 4.4-6.3 cm de largo y 2.1-2.5 cm de ancho en la subespecie mexicana, mientras que la xolocotziana tiene 4.6-6.2 cm de largo y $1.7-3 \mathrm{~cm}$ de ancho. Ápice atenuado y base redondeada. Margen dentadoserrado, con un ángulo apical agudo. Textura cartácea y glándulas presentes en toda la lámina. El pecíolo mide 1 cm de largo en ambas subespecies. Venación acródroma de posición basal con un desarrollo imperfecto (Figs. 4 a, b). La vena primaria tiene un recorrido recto no ramificado; en algunas hojas de la subespecie mexicana presentan un arco suave apical. Las venas secundarias tienen un ángulo agudo moderado con ángulo de divergencia casi uniforme, su recorrido es ligeramente curvado provisto de venas secundarias externas. Las intersecundarias son compuestas formadas por venas terciarias coalescentes muy finas, reticuladas al azar. Areolas moderadamente desarrolladas, esto es, de forma y tamaño irregular, con una disposición al azar, vénulas simples y en ocasiones ramificadas una vez (Fig. 4 c). Margen con dientes cunonioides (Fig. 4 d). Anatomía. Hoja (Fig. 5). En vista superficial, las células epidérmicas tienen paredes anticlinales sinuosas, estomas en la superficie abaxial de tipo diacítico, aunque escasamente también se presenta el tipo anomocítico. En vista transversal, en la superficie adaxial y la abaxial la cutícula es lisa, observándose 3 tipos de apéndices epidérmicos: tricomas no glandulares uniseriados de 1-4 células, con 30-112 $\mu$ m de longitud (Fig. 6 a); las glándulas pedunculadas, localizadas por encima de la cutícula, formadas por un pedicelo unicelular de 8-10 $\mu \mathrm{m}$ de largo y una cabeza redonda formada de 1-2 células (Fig. 6 b) y las glándulas secretoras, de forma bivalva, con una cabeza ancha formada de 8 células (Fig. 5 c). Epidermis simple; las células típicas son procumbentes, con dimensiones que varían de 30-58 $\mu \mathrm{m}$ x 14-28 $\mu \mathrm{m}$ en la subespecie mexicana, mientras que en la subespecie xolocotziana son de 18-58 x 8-32 $\mu \mathrm{m}$, las paredes anticlinales en ambas superficies son rectas y delgadas. El mesofilo bifacial (Figs. 5 a, b), se diferencia en empalizada y esponjoso, el parénquima en empalizada se localiza en la superficie adaxial de la hoja, esta formado por 2 estratos de células alargadas tubulares, con dimensiones que fluctúan entre los 70-104 $\mu \mathrm{m}$ x 16-26 $\mu \mathrm{m}$, ocupando $54 \%$ del mesofilo; mientras que el parénquima esponjoso está formado por 3 estratos de células organizadas laxamente, de forma irregular, ocupa 46 $\%$ del mesofilo; con gran cantidad de contenidos esféricos no determinados. Al nivel de la vena media se observa una cutícula crenada; tricomas uniseriados, glándulas secretoras bivalvas y glándulas pedunculadas en ambas superficies; las células típicas de la epidermis adaxial son cuadrangulares, las de la subespecie mexicana con 6-18 $\mu \mathrm{m} \times 8-14 \mu \mathrm{m}$, mientras que en la subespecie xolocotziana con 8-20 $\mu \mathrm{m}$ x 8-18 $\mu \mathrm{m}$, sus paredes anticlinales son rectas y gruesas, en la superficie abaxial las células típicas son de forma circular; entre ambas epidermis y próximo a la adaxial existen células de parénquima (Fig. 5 d); el floema forma un arco suave bajo el xilema, después de éste se localiza colénquima de tipo angular formado por 5-6 estratos (Fig. 5 d).

Tallo (Fig. 7). En las 2 subespecies el tallo es cuadrangular en sección transversal; en la subespecie mexicana la parte basal y media del tallo es de color morado. La cutícula es lisa, pero en los ángulos se observa crenada, con un grosor de 4-8 $\mu$ m. Se observan los 3 tipos de apéndices epidérmicos descritos para la hoja, pero los tricomas no glandulares uniseriados son abundantes en la subespecie xolocotziana, mientras que están más esparcidos y no se aprecian a simple vista en la subespecie mexicana. La epidermis es simple, células típicas de forma cuadrangular arectangular, teniendosus paredes anticlinales rectas y delgadas. Por debajo de la epidermis se distingue colénquima de tipo angular, mejor desarrollado en los ángulos de los tallos con 12-16 estratos (Fig. 7). Próximo a éste existen 3-5 capas de células de parénquima con una gran cantidad de plastidios. Hacia el interior se localiza el esclerénquima, que se organiza en bandas tangenciales hasta de 4 estratos de células, interrumpiéndose por tejido 


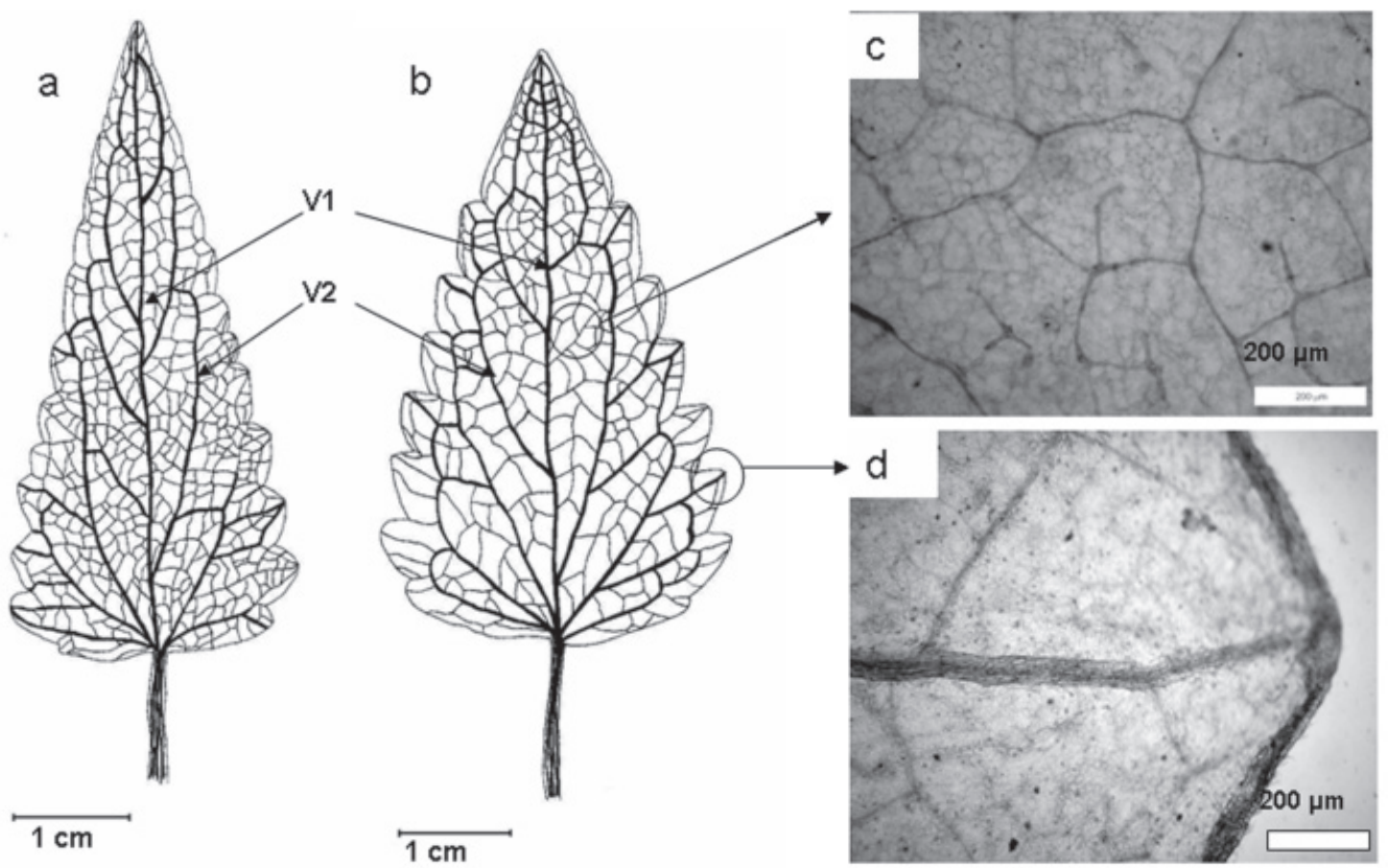

Figura 4. Hoja de Agastache mexicana con venación acródroma. a, toronjil morado ( A. mexicana ssp. mexicana); b, toronjil blanco (Agastache mexicana ssp. xolocotziana); c, areolas de desarrollo imperfecto con vénulas simples y ramificadas una vez; d, margen con diente cunonioide. Abreviaturas: V1: venas primarias y V2: venas secundarias.

parenquimático, con 30-90 $\mu \mathrm{m}$ x 23-39 $\mu \mathrm{m}$ de tamaño en la subespecie mexicana, mientras que en la subespecie xolocotziana estas bandas son de mayor dimensión, con 78-682 $\mu \mathrm{m} \times 15-54 \mu \mathrm{m}$ (Fig. 7 b). El floema secundario es más ancho en los ángulos del tallo, en la subespecie mexicana de 31-117 $\mu \mathrm{m}$ de ancho y más angosto en el resto del tallo, con 15-86 $\mu \mathrm{m}$; en la subespecie xolocotziana es de 34-64 $\mu \mathrm{m}$ en los ángulos, mientras que en el resto del tallo es de 23-40 $\mu \mathrm{m}$. El cámbium vascular está formado por 3-6 hileras de células rectangulares con paredes muy delgadas. En la parte basal del tallo, el xilema secundario está más desarrollado en la subespecie mexicana (Fig. 7 a; cuadro 1); la porosidad es difusa, los vasos son solitarios y en ocasiones agrupados en cadenas radiales de 3-4. El diámetro tangencial promedio del lumen de los vasos grandes es de $25.6 \pm 5.1 \mu \mathrm{m}(16-34 \mu \mathrm{m})$ en la subespecie mexicana y de $18.4 \pm 4.9 \mu \mathrm{m}(12-28 \mu \mathrm{m})$ en la subespecie xolocotziana. El contorno del parénquima medular es de forma circular o cuadrangular, las células son de forma isodiamétrica y tamaño homogéneo; en la subespecie mexicana no existe un canal central evidente y cuando se forma es pequeño, con diámetro de $0.78 \mathrm{~mm}$, mientras que en la xolocotziana el canal está bien desarrollado desde la base (Fig. 7 b), con un diámetro de $1.3 \mathrm{~mm}$, éste va disminuyendo hacia el ápice del tallo.
En el cuadro 1 se muestran diferencias cuantitativas de algunos caracteres anatómicos en el nivel basal, medio y superior del tallo de las 2 subespecies de A. mexicana, donde se observa que en los niveles medio y superior del tallo, existen diferencias en el tamaño de las bandas de esclerénquima, apreciándose en todos los casos mejor desarrolladas en la subespecie xolocotziana (Fig. 6c).

\section{Discusión}

Etnobotánica. El toronjil es un recurso terapéutico tradicional en el país. El registro de la información etnobotánica llevado a través de las entrevistas realizadas a los habitantes de Temoaya, Estado de México, permitió saber que el toronjil es de uso frecuente y de gran importancia medicinal en la zona. Las plantas se encuentran tanto cultivadas como silvestres y los pobladores obtienen los toronjiles del monte, de huertos o comprados en el mercado. Reconocen el toronjil morado porque presenta hojas angostas, flores y tallo de color morado y tiene un aroma dulce, mientras que el blanco lo distinguen por sus hojas anchas, flores y tallo de color blanquecino y por tener un aroma ácido (cítrico). Las amas de casa fueron las que aportaron mayor información, pues la mujer está 


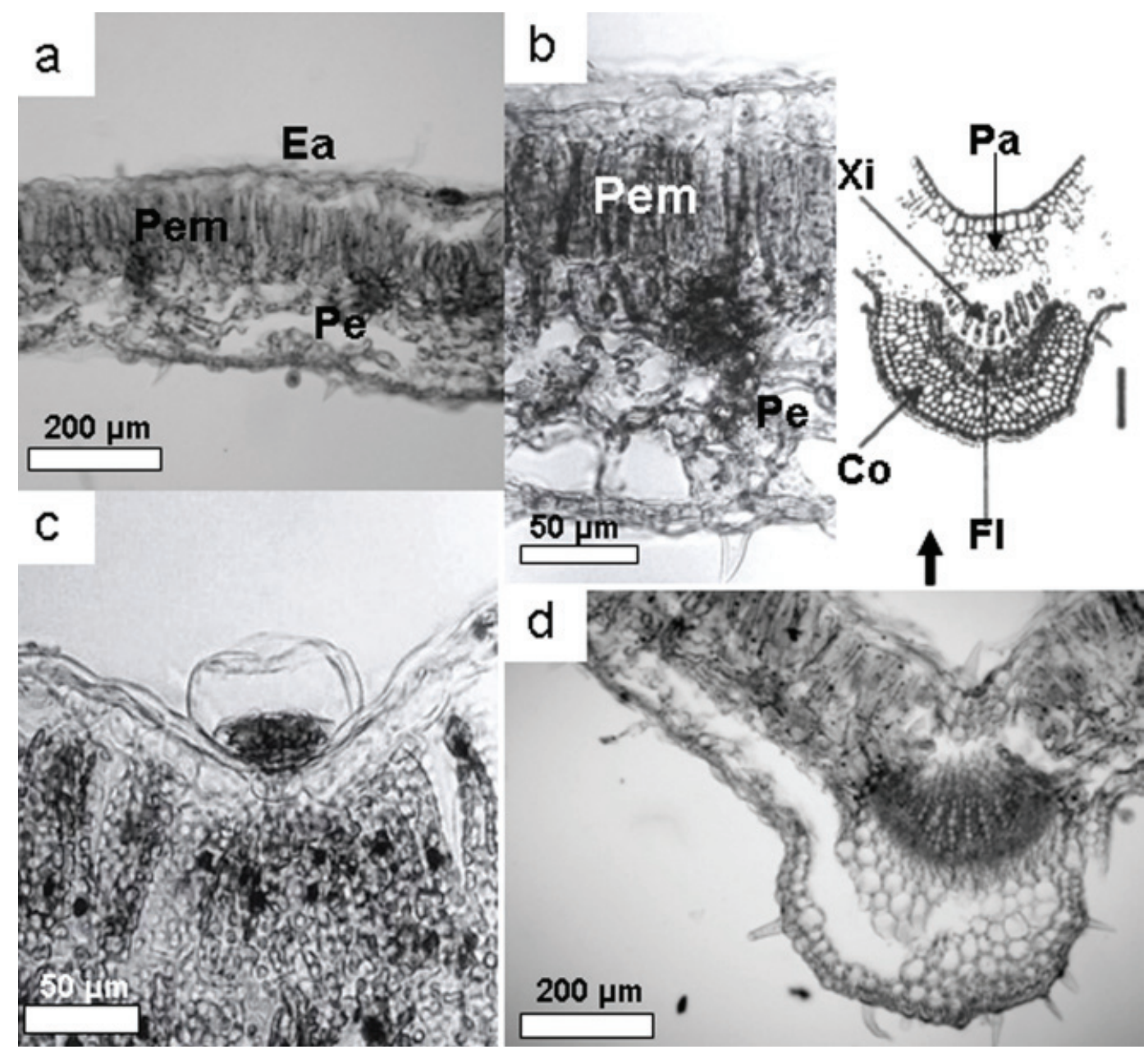

Figura 5. Características microscópicas de hoja de Agastache mexicana. a, b y d, subespecie mexicana; c, subespecie xolocotziana. Abreviaturas: $\mathrm{Ea}=$ epidermis adaxial, $\mathrm{Pem}=$ parénquima en empalizada, $\mathrm{Pe}=$ parénquima esponjoso, $\mathrm{Pa}=$ parénquima, $\mathrm{Co}=$ colénquima, $\mathrm{Fl}$ = floema primario, $\mathrm{Xi}=$ xilema primario.

considerada eje de las medidas preventivas y curativas en el espacio doméstico; es decir, como promotora de salud y curadora tradicional no especializada; siendo frecuente observar que el conocimiento y uso de las plantas medicinales recae principalmente en las mujeres, como se registra en los diferentes grupos humanos en México (Lamy y Zolla, 1978; Zolla y Mellado, 1990; Campos, 1992).

Desde la época prehispánica se tiene conocimiento sobre el uso del toronjil; se cita en diversas fuentes históricas, como el Códice De la Cruz-Badiano (1552), la obra de Francisco Hernández (1959) y el escrito de Gregorio López (López, 1580), como planta medicinal para tratar quemaduras, dolor de estómago, salpullido, para calentar las partes enfriadas, quitar tristezas y temor que procede de melancolía, entre otros. Todos estos son padecimientos que hasta nuestros días se han registrado por medio de investigaciones etnobotánicas realizadas en diferentes estados de la República Mexicana y que son tratados con el toronjil.

Aunque son diversos los usos que los habitantes de Temoaya tienen del toronjil, se menciona principalmente en la cura del susto, enfermedad considerada por Zolla et al. (1988) como un síndrome de filiación cultural o síndrome culturalmente delimitado, es decir aquellos complejos mórbidos que se perciben, clasifican y tratan conforme a claves culturales propias del grupo y en los que es evidente la apelación a procedimientos de eficacia simbólica para lograr la recuperación del enfermo. Las causas de la enfermedad del susto para la comunidad son variadas; sin embargo, se menciona frecuentemente el que pasen cosas repentinas o tener algún accidente. Esto causa tristeza, nostalgia, angustia, nerviosismo y desesperación en los enfermos, mismos síntomas que han sido registrados 


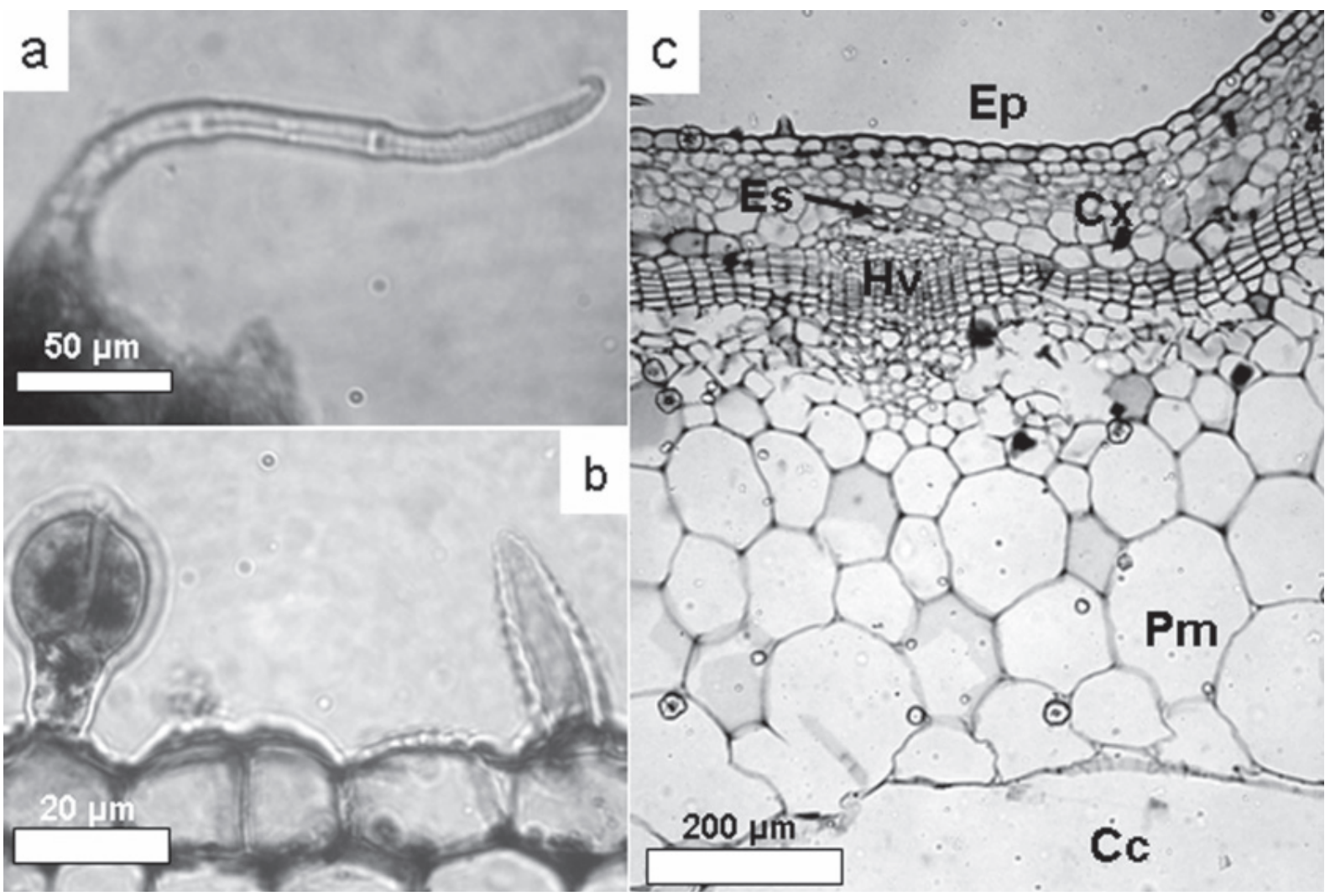

Figura 6. Características microscópicas anatómicas de hoja y tallo de Agastache mexicana. a, tricoma uniseriado en hoja de toronjil blanco; b, glándula pedunculada con cabeza de 2 células en toronjil morado; c, vista transversal del tallo de toronjil blanco. Abreviaturas: $\mathrm{Ep}=$ epidermis, Es = esclerénquima, $\mathrm{Cx}=$ córtex, $\mathrm{Pm}=$ parénquima medular, $\mathrm{Cc}=$ canal central, $\mathrm{Hv}=$ haz vascular.

Cuadro 1. Diferencias cuantitativas de algunos caracteres anatómicos obtenidos del nivel basal, medio y superior del tallo de las dos subespecies de Agastache mexicana (toronjil)

\begin{tabular}{|c|c|c|c|c|c|c|c|}
\hline \multirow[t]{2}{*}{ Tallo* } & & \multicolumn{2}{|c|}{ Nivel basal } & \multicolumn{2}{|c|}{ Nivel medio } & \multicolumn{2}{|c|}{ Nivel superior } \\
\hline & & blanco & morado & blanco & morado & blanco & morado \\
\hline \multirow{2}{*}{$\begin{array}{l}\text { Esclerénquima (Bandas) } \\
(\mu \mathrm{m})\end{array}$} & $\mathrm{L}$ & $78-682$ & $30-90$ & $117-235$ & $31-149$ & $510-667$ & $54-580$ \\
\hline & A & $15-54$ & $23-39$ & $28-36$ & $15-47$ & $23-47$ & $15-31$ \\
\hline $\begin{array}{l}\text { Diámetro tangencial de los } \\
\text { vasos del xilema }(\mu \mathrm{m})\end{array}$ & & $\begin{array}{l}18.4 \pm 4.9 \\
(12-28)\end{array}$ & $\begin{array}{l}25.6 \pm 5.1 \\
(16-34)\end{array}$ & $\begin{array}{l}24.8 \pm 3.3 \\
(18-30)\end{array}$ & $\begin{array}{l}22.1 \pm 2.9 \\
(18-28)\end{array}$ & $\begin{array}{l}24.3 \pm 4.7 \\
(16-32)\end{array}$ & $\begin{array}{l}16.4 \pm 2.7 \\
(12-22)\end{array}$ \\
\hline \multirow[t]{2}{*}{ Ancho del xilema $2^{\circ}(\mu \mathrm{m})$} & Áng & $125-172$ & $565-643$ & $188-235$ & $172-204$ & $109-157$ & $54-78$ \\
\hline & RT & $54-70$ & $455-525$ & $54-94$ & $70-86$ & $39-78$ & $23-31$ \\
\hline
\end{tabular}

*Abreviaturas: $\mathrm{L}=$ largo, A = ancho, Áng = en los ángulos, $\mathrm{RT}$ = en el resto del tallo.

por Rubel (1967) y Collado (1988) en sus investigaciones sobre el susto. A pesar de que los pobladores en Temoaya reconocen los 2 tipos de toronjil, dentro de la terapéutica tradicional no tienen alguna preferencia en su uso, pues los emplean indistintamente; registrando que la forma de preparación más frecuente es hirviendo los tallos y hojas para preparar un té que se administra oralmente y en ocasiones en agua para bañar al enfermo.

Arquitectura foliar. El estudio sobre arquitectura foliar mostró que las hojas son simples, con margen dentado 
a
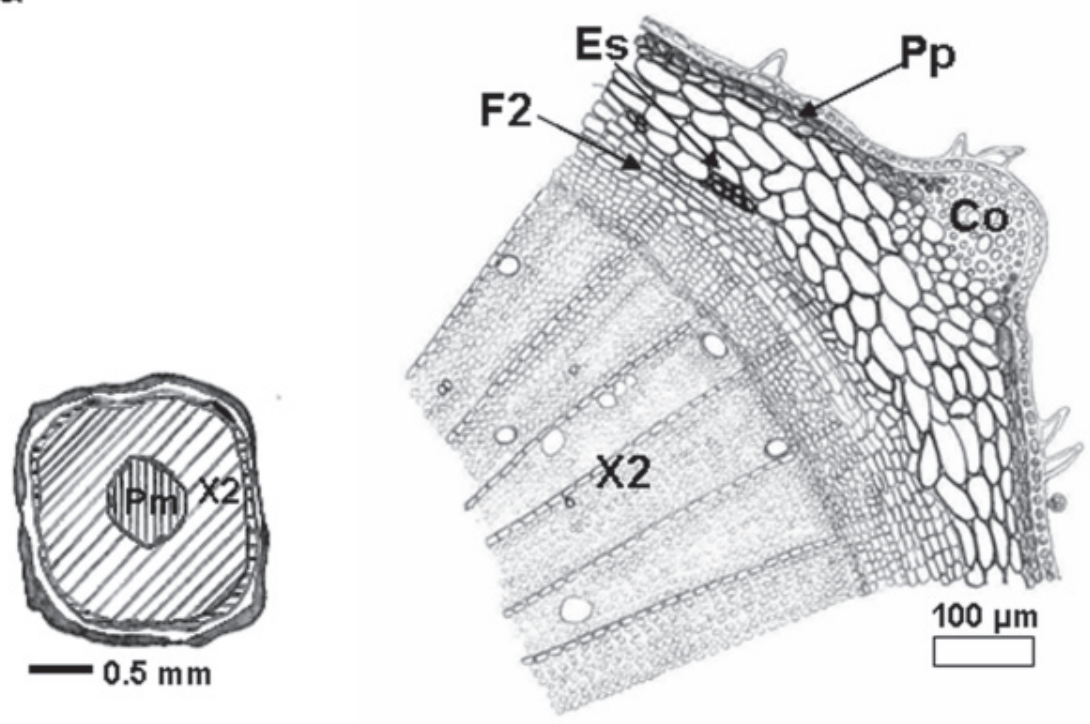

b

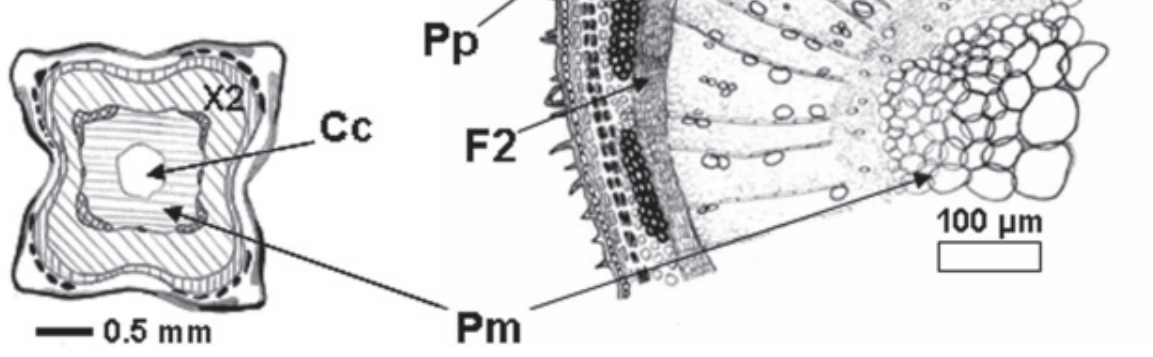

Figura 7. Sección transversal del tallo de Agastache mexicana. a, vista general y ángulo del tallo de toronjil morado, nivel basal con mayor crecimiento de xilema secundario; b, vista general y ángulo del tallo de toronjil blanco, nivel basal. Abreviaturas: $\mathrm{Co}=$ colénquima, $\mathrm{F} 2$ = floema secundario, $\mathrm{X} 2$ = xilema secundario, $\mathrm{Pm}=$ parénquima medular, $\mathrm{Pp}=$ parénquima con plastidios, Es = esclerénquima, $\mathrm{Cc}=$ canal central.

de parénquima esponjoso en las hojas y los tricomas glandulares y no glandulares, son típicos de las Lamiaceae, como lo registran Metcalfe y Chalk (1950, 1979) y Fisher (1985). Otro aspecto, es la presencia de gran cantidad de contenidos esféricos en el mesofilo de la hoja, que guardan probablemente aceites esenciales de naturaleza lipídica. Estos contenidos están bien reconocidos en las lamiáceas (Denys et al., 1991; Kuklinski, 2000); se han observado en células, en tricomas y en cavidades y canales secretores de diversos órganos vegetales, entre ellos, las hojas. En algunas especies europeas, como Melissa officinalis, los aceites esenciales se registran en los tricomas glandulares (Kuklinski, 2000). Recientemente un análisis fitoquímico comparativo de $A$. mexicana subsp. mexicana y $A$. mexicana subsp. xolocotziana reveló que la concentración y composición química de los

y diente cunonioide, siguen el patrón general descrito para las Lamiales (Hickey y Wolfe, 1975). Las areolas de desarrollo imperfecto con disposición al azar, se describen por primera vez para A. mexicana; por lo que la arquitectura foliar no mostró diferencias evidentes entre las 2 subespecies.

Anatomía. El estudio de los caracteres microscópicos en las 2 subespecies de toronjil reveló que la forma cuadrangular y la gran cantidad de colénquima en los ángulos de los tallos, así como los estomas de tipo diacítico y el mesofilo bifacial formado por 2 estratos de parénquima en empalizada, 3 monoterpenos, registrados ampliamente en el género Agastache, varían en cuanto a su estructura química y composición entre las 2 subespecies (Estrada-Reyes et al., 2004). En el tallo existen algunas características anatómicas que muestran diferencias entre ambos toronjiles. Una de ellas son los tricomas no glandulares uniseriados que se presentan en mayor cantidad y son evidentes a simple vista en la subespecie xolocotziana, a diferencia de la subespecie mexicana, en la que los tricomas son escasos y se requiere de un lente de aumento para observarlos. A pesar de que Metcalfe y Chalk (1979) establecen que la 
abundancia de los tricomas no es un criterio taxonómico y que sólo la presencia y el tipo de apéndices epidérmicos son importantes en la caracterización de las familias, la cantidad de tricomas puede ser un carácter diagnóstico importante en la diferenciación en el nivel de especie o por debajo de ésta. Las bandas de esclerénquima que se disponen en el córtex de los tallos de ambos toronjiles varían en tamaño, siendo mayores en el toronjil blanco. De acuerdo con Metcalfe y Chalk (1979) estas bandas difieren en forma y estructura entre diversas especies de lamiáceas, por lo que pueden emplearse como un carácter diagnóstico. En el xilema secundario que se desarrolla en la parte basal del tallo hay diferencias, observándose que en el toronjil morado el diámetro de los vasos y la cantidad de xilema secundario es mayor que en el toronjil blanco; independientemente del tamaño de las plantas, estos contrastes fueron constantes entre ambas subespecies.

Este estudio nos permitió conocer que los toronjiles siguen siendo un recurso herbolario de gran importancia para los habitantes de Temoaya, principalmente empleado en la cura del susto, padecimiento que es atendido solamente por los practicantes de la medicina tradicional popular. Por esta razón, acordamos con Warren y Raffa (1987) y con Collado (1988) en considerar que el éxito de la medicina occidental puede darse sólo en la medida en que se establezca un plan de comunicación recíproca y una compatibilidad con las creencias y prácticas etnomédicas, así como dejar de considerar el "susto" con indiferencia o como una curiosidad folklórica, puesto que es una situación patológica que afecta integralmente al individuo y que amenaza su vida.

Es de resaltar el conocimiento que los habitantes de Temoaya, otomíes y mestizos, tienen sobre la morfología de los toronjiles, reconociéndolos por la forma de la hoja, color de la flor y tallo, así como por su aroma. Respecto al estudio anatómico, los caracteres que contribuyen a separar ambas subespecies se observan en el nivel basal del tallo y son los tricomas no glandulares uniseriados en mayor proporción, bandas de esclerénquima mejor desarrolladas (78-682 $\mu \mathrm{m}$ X 15-54 $\mu \mathrm{m}$ ), el diámetro más pequeño de los vasos $(18.4 \mu \mathrm{m} \pm 4.9 \mu \mathrm{m})$, el xilema secundario poco desarrollado $(54-70 \mu \mathrm{m})$ y un canal central de mayor diámetro (1.3 mm) en la subespecie xolocotziana los que la distinguen de la subespecie mexicana. Sin embargo, en los niveles medio y superior del tallo, la diferencia notable entre ambas se limita al tamaño de las bandas de esclerénquima, apreciándose mejor desarrolladas en xolocotziana. Por lo que este estudio comparativo se suma a las diferencias encontradas en el análisis fitoquímico (Estrada-Reyes et al., 2004), y los estudios farmacológico (Contreras, 1987; Espíritu, 1991; Aguirre, 2003) y de propagación (Chávez, 1986). Las características anatómicas de ambos toronjiles aportan una herramienta diagnóstica útil para diferenciarlos, quedando así de manifiesto la importancia y utilidad de la anatomía vegetal para verificar la autenticidad del material botánico medicinal cuando se comercializa. Se considera que la investigación etnobotánica y anatómica obtenida de estas subespecies aporta información de gran interés para la Farmacopea Herbolaria de los Estados Unidos Mexicanos.

\section{Agradecimientos}

A la M. en C. María Patricia Jácquez Ríos, del Herbario de la Facultad de Estudios Superiores Iztacala, UNAM, por la revisión crítica del manuscrito y a los revisores anónimos que con sus valiosas sugerencias mejoraron sustancialmente este trabajo.

\section{Literatura citada}

Aguilar, A. y J. R. Camacho. 1985. Uso popular de las plantas medicinales y su distribución por aparatos y sistemas. Archivos de investigación médica. México, D. F. Suplemento 6:13-14,

Aguilar, A., J. R. Camacho, S. Chino, P. Jácquez y M. E. López. 1994. Herbario medicinal del Instituto Mexicano del Seguro Social. IMSS, México, D. F. 253 p.

Aguilar-Rodríguez, S. 1998. Técnicas de laboratorio para el estudio de las embriófitas. In Plantae. Introducción al estudio de las plantas con embrión, segunda edición, J. D. Tejero D. y M. P. Granillo V. (eds.). Universidad Nacional Autónoma de México, Campus Iztacala, Estado de México. p. $247-285$.

Aguirre, H. E. 2003. Estudio fitoquímico y farmacológico de Agastache mexicana ssp. mexicana y Agastache mexicana ssp. xolocotziana. Tesis maestría, Colegio de Postgraduados, Montecillo. Texcoco, Estado de México. 121 p.

Arzate, B. J. 1999. Temoaya. Monografía municipal. Instituto Mexiquense de Cultura-AMECROM. Toluca, Estado de México. 143 p.

Campos, N. R. 1992. Prácticas médicas populares. Algunas experiencias sobre el proceso de autoatención curativa. In La antropología médica en México, tomo1,R. Campos N. (ed.). Universidad Autónoma Metropolitana e Instituto Mora, México, D. F. p.186-210.

Campos, N. R. 1997. Nosotros los curanderos. Nueva Imagen, México, D. F. 316 p.

Cervantes, E. M. T. 1978. Estructura ocupacional y organizacional social de una comunidad otomí del Estado de México, San Pedro Arriba, Temoaya. Tesis maestría en etnología (especialidad antropología social), Instituto Nacional de Antropología e Historia, Secretaría de Educación Pública, México, D. F. 117 p.

Chávez, C. C. Y. 1986. Propagación vegetativa de toronjil morado (Agastache mexicana (HBK) Lint \& Epling) y 
toronjil blanco (Agastache sp.) por esquejes de tallo, bajo condiciones de invernadero. Tesis, Facultad de Ciencias, Universidad Nacional Autónoma de México. México, D. F. $127 \mathrm{p}$.

Collado, A. R. 1988. Una enfermedad paralela: "el susto". Tesis doctorado (Psicología social) Facultad de Psicología, Universidad Nacional Autónoma de México, México, D. F. $241 \mathrm{p}$.

Contreras, T. B. 1987. Metabolitos secundarios aislados de toronjil blanco (Agastache sp.). Tesis, Escuela QFB, Universidad Femenina de México, México D. F. 72 p.

Cotton, M. C. 1998. Ethnobotany, principles and aplications. Wiley, New York. 424 p.

Curtis, P. J. 1986. Microtecnia vegetal. Trillas, México, D. F. $106 \mathrm{p}$.

De la Cruz, M. y J. Badiano. 1552. Libellus de medicinalibus indorum herbis, edición facsimilar 1991. Fondo de Cultura Económica, Instituto Mexicano del Seguro Social. México, México, D. F. 258 p.

Denys, J. Ch., J. E. Simon y M. P. Widrlechner. 1991. Characterization of essential oil of Agastache species. Journal of Agricultural and Food Chemistry 39:19461949.

Espíritu, C. L. P. 1991. Estudio quimiotaxonómico comparativo entre el toronjil rojo y el toronjil blanco (sp.) Tesis, Escuela de Psicología, Universidad Femenina de México. México, D. F. 65 p.

Estrada-Reyes, R., E. Aguirre Hernández, A. García-Argáez, M. Soto Hernández, R. Bye, E. Linares, G. Heinze y M. Martínez-Vázquez. 2004. Comparative chemical composition of Agastache mexicana subsp. mexicana and A. mexicana subsp. xolocotziana. Biochemical Systematics and Ecology 32:685-694.

Fahn, A. 1982. Anatomía vegetal. Blume, Madrid. 599 p.

FHEUM. 2001. Farmacopea Herbolaria de los Estados Unidos Mexicanos. Secretaría de Salud, México, D. F. 228 p.

Fisher, D. G. 1985. Morphology and anatomy of the leaf of Coleus blumei (Lamiaceae). American Journal of Botany 72:392-406.

Galindo, M. Y. 1982. Estudio farmacológico de algunas plantas medicinales reportadas popularmente por la población mexicana para el tratamiento de padecimientos cardiovasculares. Tesis, Escuela Nacional de Estudios Profesionales Iztacala, Universidad Nacional Autónoma de México. Estado de México. 100 p.

García, E. 2004. Modificaciones al sistema de clasificación climática de Köppen. Serie Libros 6. Instituto de Geografía,Universidad Nacional Autónoma de México. México, D. F. 90 p.

Hernández, F. 1959. Historia natural de Nueva España, vol. 1, Obras completas, tomo II. Universidad Nacional Autónoma de México, México, D.F.

Hernández-Xolocotzi, E. 1979. El concepto de etnobotánica. In La etnobotánica: tres puntos de vista y una perspectiva, A. Barrera (ed.). Instituto de Investigaciones sobre Recursos Bióticos, México, D. F. p. 13-18.

Hickey, L. J. 1973. Classification of architecture of dicotyledonous leaves. American Journal of Botany 60:17-33.
Hickey, L. J. 1974. Arquitectura de las hojas dicotiledóneas. Boletín de la Sociedad Argentina de Botánica16:1-26.

Hickey, L. J. y J. A. Wolfe. 1975. The bases of angiosperm phylogeny. Vegetative morphology. Annals of Missouri Botanical Garden 62:538-589.

IAWA Committee. 1989. IAWA list of microscopic features for hardwood identification. International Association of Wood Anatomist. Bulletin new series10:219-232.

Instituto Nacional Indigenista. 1995. Etnografía contemporánea de los pueblos indígenas de México: Región Centro. Instituto Nacional Indigenista, Secretaría de Desarrollo Social, México, D. F. 384 p.

Kuklinski, C. 2000. Farmacognosia. Estudio de las drogas y sustancias medicamentosas de origen natural. Omega, Barcelona. 515 p.

Lamy, P. y C. Zolla. 1978. La etnobotánica en relación con los problemas de la salud en México. IMEPLAM. Medicina Tradicional 5:1-11.

López, G. 1580. Tesoro de medicinas para diversas enfermedades, edición facsimilar 1990. Instituto Mexicano del Seguro Social, Instituto Nacional de Antropología e Historia, México, D. F.

Lot, A. y F. Chiang. 1986. Manual de Herbario: Administración y manejo de colecciones, técnicas de recolección y preparación de ejemplares botánicos. Instituto de Biología. Universidad Nacional Autónoma de México. Consejo Nacional de la Flora de México. A. C. México, D. F. 142 p.

Martínez, A. M. A. 1976. Posible metodología a seguir en el estudio de las Plantas medicinales mexicanas. In Estudios sobre etnobotánica y antropología médica, T. C. Viesca,(ed.). IMEPLAM, México, D. F. p. 75-83.

Martínez, A. M. A. 1994. Estado actual de las investigaciones etnobotánicas en México. Boletín de la Sociedad Botánica de México 55:65-74.

Medina, G. J. L. 1998. Cronología de la erupción dentaria en escolares de Temoaya, Estado de México. Tesis, Facultad de Estudios Superiores Zaragoza, Universidad Nacional Autónoma de México, México, D. F. 62 p.

Metcalfe, C. R. y L. Chalk. 1950. Anatomy of the dicotyledons. Leaves, stem, and wood in relation to taxonomy with notes on economic uses, vol. II. Clarendon, Oxford. p. 1041- 1053.

Metcalfe, C. R. y L. Chalk. 1979. Anatomy of the dicotyledons. Systematic anatomy of leaf and stem, with a brief history of the subject, vol. I. Clarendon, Oxford. 294 p.

Murillo, J. A. 2001. Patrones de arquitectura foliar en la subtribu Conceveibinae (Euphorbiaceae). Caldasia 23:155-162.

Rubel A. J. 1967. El susto en Hispanoamérica. América indígena. Instituto Indigenista Interamericano 27:70-89 (México).

Rzedowski, J. 1988. Vegetación de México. Limusa, México, D. F. 432 p.

Toledo, V. 1995. New paradigms for a new ethnobotany. Reflections on the case of México. In Evolution of a discipline, R. S. Evans y R. S. Von (eds.). Dioscorides, Portland. p.79-92.

Triplett, J. K. y B. K. Kirchoff. 1991. Lamina architecture and anatomy in the Heliconiaceae and Musaceae (Zingiberales). Canadian Journal of Botany 69:887-900.

Warren, P. y A. Raffa. 1987. Medicina tradicional y moderna 
entre los achuar del río Huasaga. Hombre y Ambiente, el punto de vista indígena 1:91-94.

Zolla, C., S. del Bosque, A. Tascón, V. Mellado y C. Maqueo. 1988. Medicina tradicional y enfermedad. Centro Interamericano de Estudios de Seguridad Social - Instituto
Mexicano del Seguro Social. México, D. F. 146 p.

Zolla, C. y V. Mellado. 1990. Mujer y salud. La función de la medicina doméstica en el mundo rural mexicano. Programa Interdisciplinario de Estudios de la Mujer - Colegio de México, México, D. F. 33 p. 